\title{
Universal Nonadiabatic Control of Small-Gap Superconducting Qubits
}

\author{
Daniel L. Campbell, ${ }^{1, \dagger+}$ Yun-Pil Shim $\odot,{ }^{2,3, \S}$ Bharath Kannan $\odot,{ }^{1,4}$ Roni Winik, ${ }^{1}$ David K. Kim, ${ }^{5}$ Alexander Melville, ${ }^{5}$ \\ Bethany M. Niedzielski, ${ }^{5}$ Jonilyn L. Yoder, ${ }^{5}$ Charles Tahan, ${ }^{2}$ Simon Gustavsson, ${ }^{1}$ and William D. Oliver $\circledast^{1,4,5,6, *}$ \\ ${ }^{1}$ Research Laboratory of Electronics, Massachusetts Institute of Technology, \\ Cambridge, Massachusetts 02139, USA \\ ${ }^{2}$ Laboratory for Physical Sciences, College Park, Maryland 20740, USA \\ ${ }^{3}$ Department of Physics, University of Maryland, College Park, Maryland 20740, USA \\ ${ }^{4}$ Department of Electrical Engineering and Computer Science, Massachusetts Institute of Technology, \\ Cambridge, Massachusetts 02139, USA \\ ${ }^{5}$ MIT Lincoln Laboratory, 244 Wood Street, Lexington, Massachusetts 02420, USA \\ ${ }^{6}$ Department of Physics, Massachusetts Institute of Technology, Cambridge, Massachusetts 02139, USA
}

(Received 31 March 2020; revised 5 October 2020; accepted 7 October 2020; published 14 December 2020)

\begin{abstract}
Resonant transverse driving of a two-level system as viewed in the rotating frame couples two degenerate states at the Rabi frequency, an equivalence that emerges in quantum mechanics. While successful at controlling natural and artificial quantum systems, certain limitations may arise (e.g., the achievable gate speed) due to nonidealities like the counterrotating term. We introduce a superconducting composite qubit (CQB), formed from two capacitively coupled transmon qubits, which features a small avoided crossing - smaller than the environmental temperature-between two energy levels. We control this low-frequency CQB using solely baseband pulses, nonadiabatic transitions, and coherent LandauZener interference to achieve fast, high-fidelity, single-qubit operations with Clifford fidelities exceeding 99.7\%. We also perform coupled qubit operations between two low-frequency CQBs. This work demonstrates that universal nonadiabatic control of low-frequency qubits is feasible using solely baseband pulses.
\end{abstract}

DOI: 10.1103/PhysRevX.10.041051

Subject Areas: Quantum Information

\section{INTRODUCTION}

Variations on the transmon qubit [1] and the capacitively shunted flux qubit [2] form the foundation for contemporary superconducting quantum computing [3-7] and explorations of quantum mechanics in solid-state systems. In the context of quantum control, we generally view superconducting qubits as "artificial atoms": electrical circuits that exhibit quantum states and energy levels similar, in many respects, to those present in natural atoms. It is then a straightforward extension to use a resonant, transverse

\footnotetext{
* Corresponding author.

william.oliver@mit.edu

Corresponding author. daniel.campbell.22@us.af.mil

*Present address: Air Force Research Laboratory, Information Directorate. 585 Brooks Rd. Rome, New York, 13441 USA.

${ }^{\S}$ Present address: Department of Physics, University of Texas at El Paso, El Paso, Texas 79968, USA

Published by the American Physical Society under the terms of the Creative Commons Attribution 4.0 International license. Further distribution of this work must maintain attribution to the author(s) and the published article's title, journal citation, and DOI.
}

field - typically at microwave frequencies - to drive transitions between states and thereby perform qubit operations. For superconducting qubits [8], with their large electric or magnetic dipole moments, this approach has worked remarkably well, enabling single-qubit gate fidelities that exceed 99.9\% and two-qubit fidelities that are not far behind $[7,9,10]$. However, as architectures scale and qubit numbers increase, it becomes increasingly challenging to route microwave control signals in higher-density circuits while avoiding unwanted cross talk. Reducing the qubit frequency - and thereby the resonant drive frequencyhelps mitigate capacitive cross talk but at the expense of the achievable Rabi frequency (gate speed) before nonidealities, like the counterrotating term, come into play. Furthermore, for qubit frequencies below the environmental temperature, one may question whether such an operation is even practically feasible due to the excess excited-state population in equilibrium and the resulting need for fast gates to polarize (initialize) the qubit in its ground state.

Spin-based qubits in semiconductors offer an alternative path forward. Resonant single-qubit operations based on magnetic-field driving of spin qubits are relatively slow, necessitating large driving amplitudes [11,12] and, in conjunction with the relatively small qubit size (high qubit density), result in excessive microwave cross talk. 
(a)

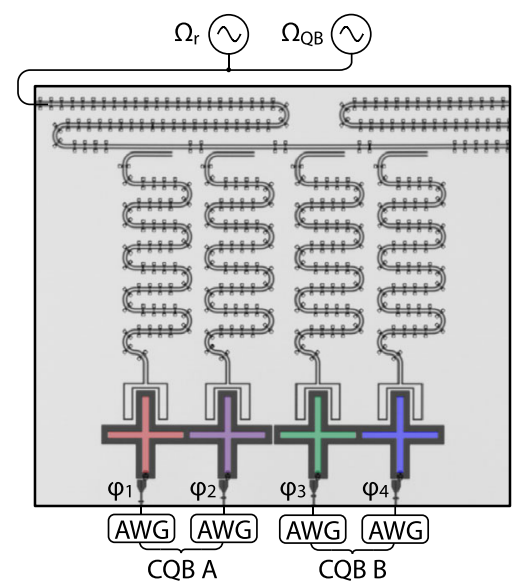

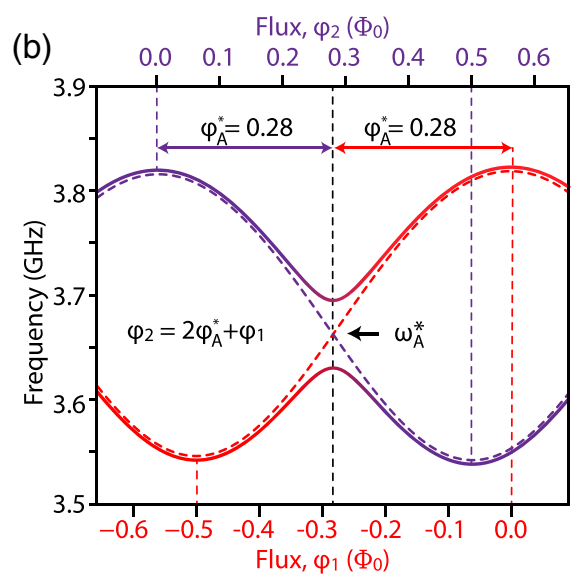

(c)

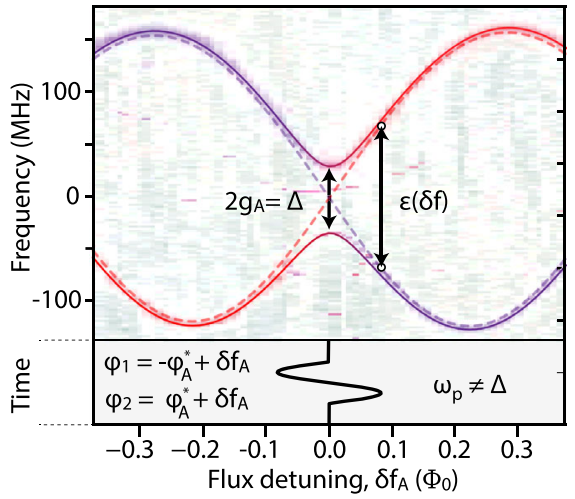

FIG. 1. Device and control. (a) Optical micrograph of two composite qubits (CQB-A and CQB-B) comprising four transmon qubits (1, 2,3 , and 4) with nearest-neighbor capacitive coupling. A microwave feedline allows frequency multiplexed readout and state preparation via the microwave driving fields $\Omega_{\mathrm{r}}$ and $\Omega_{\mathrm{QB}}$, respectively. (b) Eigenenergies of individual (dashed lines) and coupled (solid lines) asymmetric-junction transmons 1 and 2 (CQB-A). An avoided level crossing occurs when the coupled transmons are resonantly biased at $\varphi_{1,2}= \pm \varphi_{\mathrm{A}}^{*}$. (c) Upper panel: corresponding measured excited-state spectroscopy centered at zero frequency (offset in frequency) to form a two-level system model with parameters $\Delta$ and $\varepsilon(\delta f)$. Near the avoided crossing region, $\varepsilon$ is proportional to the flux detuning $\delta f$, realized by simultaneously biasing $\varphi_{1}$ and $\varphi_{2}$. Lower panel: nonadiabatic control implemented by applying a single period of a nonresonant $\left(\omega_{p} \neq \Delta\right)$ sinusoidal excursion about the avoided crossing.

Consequently, from the very beginning, the spin-qubit community has instead largely relied on effective, encoded qubits comprising two or more individual spins and their exchange interactions [13]. The exchange interaction enables fast encoded qubit gates controlled solely using baseband pulses, alleviating the need for pulsed-microwave control signals, whose shortcomings include expense, speed limitations associated with qubit anharmonicity, and frequency-dependent cross-talk compensation. Additionally, the qubit encoding features a degree of immunity to global field fluctuations $[14,15]$. This physics, which occurs naturally for spin systems and was in fact used in several early demonstrations with superconducting charge qubits [16-18], motivates us to explore analogous forms of encoding and quantum control for small-gap superconducting systems [19].

\section{EXPERIMENTAL SETUP}

In this work, we demonstrate universal control of a superconducting composite qubit (CQB) using solely baseband pulses reliant on nonadiabatic, Landau-Zener transitions and quantum interference [20-22]. The CQB comprises two coupled transmon qubits and features a gap $(\Delta / 2 \pi \approx 65 \mathrm{MHz})$ that is appropriately sized for such baseband control. The small gap reduces the relaxation rate in the computational basis [2,20], and the composite nature of the CQB design features resilience to both environmental flux noise [23-25] and photon shot noise from the readout resonator [26-28]. We present a tune-up protocol for single-CQB and two-CQB gates and benchmark their performance, achieving $99.7 \%$ single-qubit average
Clifford fidelity. Although demonstrated with CQBs, the nonadiabatic control protocols demonstrated here are generally applicable to quantum systems featuring small gaps.

\section{A. Device description}

Our test device comprises four asymmetric superconducting transmon qubits [29] of the "xmon" geometry [30] with fixed, nearest-neighbor capacitive coupling [Fig. 1(a)]. Pairs of transmons are grouped to form the composite qubits used here, denoted "CQB-A" and "CQB-B." Qubit spectroscopy of CQB-A [Fig. 1(b)] shows the constituent transmon spectra of the ground-state $\left|g_{i}\right\rangle$ to excited-state $\left|e_{i}\right\rangle$ transitions for $i=1,2$ as a function of the reduced flux biases $\varphi_{i} \equiv \Phi_{i} / \Phi_{0}$, where $\Phi_{i}$ is the magnetic flux and $\Phi_{0}$ is the superconducting flux quantum. Similar spectra are observed for CQB-B and transmons $i=3,4$ [31].

When the transmons are biased at the same frequency, $\omega_{1}=\omega_{2} \equiv \omega_{\mathrm{A}}^{*}$, an avoided crossing $\Delta=2 g_{\mathrm{A}}$ opens due to the fixed capacitive coupling within CQB-A of strength $g_{\mathrm{A}}$. The size of the avoided crossing, $\Delta / 2 \pi \approx 65 \mathrm{MHz}$, is determined predominantly by the value of the coupling capacitance, but its location-centered at frequency $\omega_{\mathrm{A}}^{*}\left(\varphi_{\mathrm{A}}^{*}\right)$ - can generally be chosen along the transmon spectra at flux biases $\varphi_{1,2}=\mp \varphi_{\mathrm{A}}^{*}$. In Fig. 1(b), we have chosen $\varphi_{\mathrm{A}}^{*}=0.28$. More generally, CQB-A can be flux biased over its entire frequency range using the individual transmon biases $\varphi_{1}$ and $\varphi_{2}=2 \varphi_{\mathrm{A}}^{*}+\varphi_{1}$, and similarly for CQB-B and its transmons.

The CQB subspace, given by the avoided crossing in Fig. 1(c), is described by the standard two-level-system Hamiltonian 


$$
\hat{H} / \hbar=-\frac{1}{2}\left[\Delta \hat{\sigma}_{z}+\varepsilon \hat{\sigma}_{x}\right]
$$

where $\hbar=h / 2 \pi$ (with $h$ being Planck's constant) and $\hat{\sigma}_{x}$ and $\hat{\sigma}_{z}$ are Pauli operators. For highly asymmetric transmons, the parameter $\varepsilon=2 \delta \omega \sin \left(2 \pi \varphi_{\mathrm{A}}^{*}\right) \sin \left(2 \pi \delta f_{\mathrm{A}}\right)$ is the difference between the bare transmon frequencies referenced with respect to the avoided crossing through the flux detuning $\delta f_{\mathrm{A}} \equiv \varphi_{1,2} \mp \varphi_{\mathrm{A}}^{*}$, with $\delta \omega$ defined as the difference between maximum and minimum transmon frequencies [32]. Near the avoided crossing, $\varepsilon \approx 4 \pi \delta \omega \sin \left(\varphi_{\mathrm{A}}^{*}\right) \delta f_{\mathrm{A}}$ is approximately a linear function of $\delta f_{\mathrm{A}}$, reminiscent of the persistent current flux qubit [33,34] (see also Supplemental Material [31]). Although $\Delta$ transversally couples the bare (diabatic) transmon states, we have elected to associate $\Delta$ with $\hat{\sigma}_{z}$, as the computational basis $\{|0\rangle,|1\rangle\}$ is defined at the avoided crossing. At this bias point, the coupling hybridizes the bare transmon states to form the CQB computational states $|0\rangle,|1\rangle=$ $\left|g_{1}, e_{2}\right\rangle \pm\left|e_{1}, g_{2}\right\rangle$.

\section{B. Initialization and readout}

Initializing the $\mathrm{CQB}$ in state $|0\rangle$ does not require a precise knowledge of $\omega_{A, B}^{*}$ or microwave mixer calibration. Beginning with both transmons in their ground states $\left|g_{1}, g_{2}\right\rangle$, the CQB is biased far from $\varepsilon=0$ (transmon frequency degeneracy). Then, in the presence of a continuous-wave microwave drive, the system is further detuned such that one of the transmons (e.g., transmon 1) adiabatically passes through the resonance with the drive, which excites the CQB to the diabatic state $\left|e_{1}, g_{2}\right\rangle$. The field is then turned off, and the CQB is adiabatically ramped back to the degeneracy point, initializing the qubit in state $|0\rangle$ [31].

CQB readout is performed by adiabatically detuning the CQB away from the avoided crossing, such that the hybridized computational states $|0\rangle$ and $|1\rangle$ are uniquely mapped onto the bare (diabatic) transmon states $\left|e_{1}, g_{2}\right\rangle$ and $\left|g_{1}, e_{2}\right\rangle$. Doing so enables CQB readout using standard dispersive readout on the underlying transmons [31]. As we describe in the discussion surrounding Fig. 3, although dispersive transmon measurement performed at degeneracy cannot distinguish CQB states $|0\rangle$ and $|1\rangle$, it has the useful property that it can be used to detect leakage out of the $\mathrm{CQB}$ subspace without destroying the CQB quantum information.

\section{UNVIERSAL NONADIABATIC GATES WITH COMPOSITE QUBITS}

When applied to small-gap qubits, resonant excitation in the perturbative Rabi-driving regime $\varepsilon(\delta f) \ll \Delta$ results in nutation periods $\tau \gg 1 / \Delta \approx 15 \mathrm{~ns}$ and leads to prohibitively slow qubit gates. A better approach is to use a nonresonant baseband pulse that sweeps the parameter $\varepsilon$ of a qubit around and through a transverse avoided crossing of size $\Delta$. For sufficiently large driving amplitudes $\varepsilon\left(\delta f_{j}\right)>\Delta$, these excursions cause coherent, nonadiabatic transitions, which in conjunction with quantum interference, lead to controllable state transitions on a timescale that can approach the speed limit for the system, $\tau \sim 1 / \Delta$. This effect, known as Landau-Zener-Stueckelberg interference, has been demonstrated in both natural and artificial atomic systems [22], including demonstrations of Stückelberg interferometry [20,21,35], qubit cooling [36], amplitude spectroscopy [37], temporal oscillations $[37,38]$, and its use in the quantum simulation of universal conductance fluctuations [39] and weak localization [32]. In this "strong-driving" regime, the trajectory of the Bloch vector is no longer a simple function of the amplitude, frequency, or phase of a sinusoidal drive (as it is in the Rabi-driving case), necessitating an alternative gatecalibration protocol. Our approach begins with the CQB prepared in state $|0\rangle$ at the avoided crossing, where it is first-order protected from flux noise [23], and we use a single-period sinusoidal pulse to implement quantum control [see Fig. 1(c)].

\section{A. Single composite qubit gates}

To gain intuition, we first note that a large-amplitude, solely diabatic excursion away from the avoided crossing effectively performs a 50:50 beamsplitting operation, projecting state $|0\rangle$ onto an equal superposition of the diabatic states $\left|g_{1}, e_{2}\right\rangle$ and $\left|e_{1}, g_{2}\right\rangle$ (dashed lines). Away from the avoided crossing, the higher-energy diabatic state accrues a relative azimuthal phase at a rate proportional to the energy separation $\varepsilon(\delta f)$. Rapidly returning to the avoided crossing region, we perform a second "beamsplitter"-type operation, which again mixes the states, resulting in a general superposition state $\alpha|0\rangle+\beta|1\rangle$ depending on the accrued phase and quantum interference. This case is conceptually similar to the Larmor control of early charge qubits [16-18]. In those experiments, a qubit starting in a diabatic state far from its avoided crossing was rapidly pulsed to the avoided crossing region, where it underwent Larmor precession and was then rapidly returned to its starting point.

In practice, we use one period of a finite amplitude sinusoid [Fig. 1(c)], $\delta f=A_{p} \sin \left(\omega_{p} t\right)$, that features partially diabatic excursions and incorporates the mixing and quantum interference associated with leaving, traversing, and returning to the avoided crossing region. Because of the proximity to the avoided crossing, $\varepsilon$ is proportional to $\delta f$, and we can similarly parametrize $\varepsilon=\varepsilon_{p} \sin \left(\omega_{p} t\right)$ without loss of generality. The symmetric driving protocol has the added benefit of canceling dc components associated with pulse transients, creating a "dynamic sweet spot." Although this driving protocol does not likewise protect a CQB from the direct flux control cross talk of another CQB, the fact that the pulses are essentially in the quasistatic limit results 


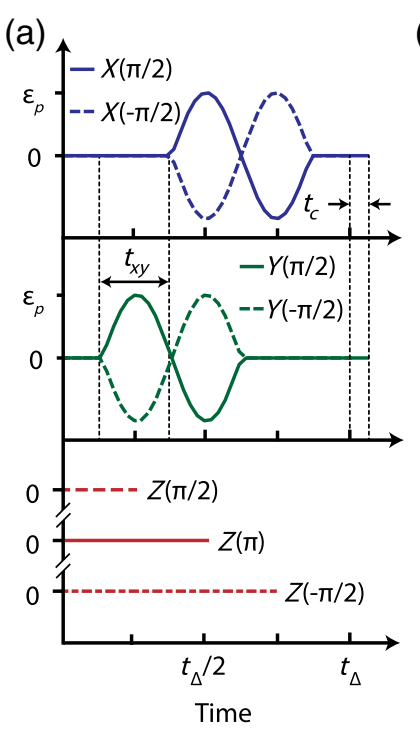

(b)

\begin{tabular}{ccccc} 
Key & Gate & Amp. & $t_{x y}$ & Duration \\
\hline \hline & $X(\pi / 2)$ & $\varepsilon_{p}$ & 0 & $t_{\Delta}+t_{c}$ \\
$\square$ & $X(-\pi / 2)$ & $-\varepsilon_{p}$ & 0 & $t_{\Delta}+t_{c}$ \\
$\square$ & $Y(\pi / 2)$ & $\varepsilon_{p}$ & $-t_{\Delta} / 4$ & $t_{\Delta}+t_{c}$ \\
$\square$ & $Y(-\pi / 2)$ & $-\varepsilon_{p}$ & $-\mathrm{t}_{\Delta} / 4$ & $t_{\Delta}+t_{c}$ \\
$\square$ & $Z(\pi / 2)$ & & & $t_{\Delta} / 4$ \\
$\square$ & $Z(\pi)$ & & & $2 t_{\Delta} / 4$ \\
$\square$ & $Z(-\pi / 2)$ & & $3 t_{\Delta} / 4$ \\
$\mathrm{CQB}$ & $\varepsilon_{p}(\mathrm{MHz})$ & $t_{\Delta}(\mathrm{ns})$ & $t_{c}(\mathrm{~ns})$ \\
\hline \hline A & 81.2 & 15.58 & 0.77 \\
B & 76.0 & 14.27 & 0.63 \\
& & &
\end{tabular}

(c)

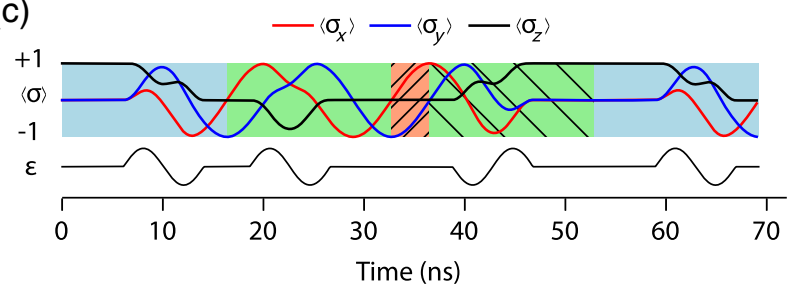

(d)

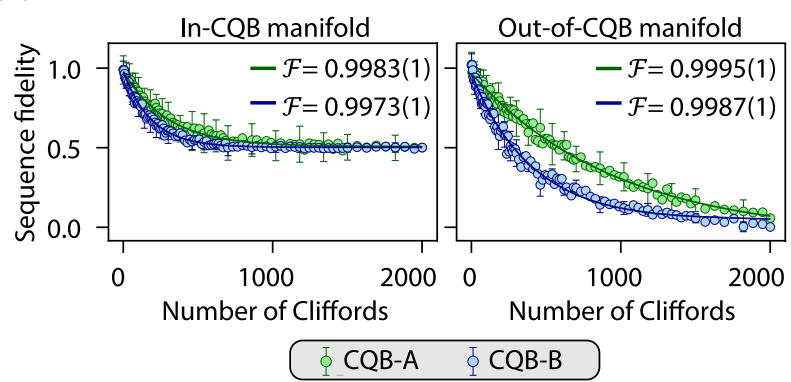

FIG. 2. Single-CQB gates. (a) Pulses for $X, Y$, and $Z$ gates. For $X( \pm \pi / 2)$ and $Y( \pm \pi / 2)$ gates, the sinusoidal pulse is applied within a time window with a relative shift $t_{x y}=t_{\Delta} / 4=2 \pi / 4 \Delta$, which establishes the $x$ and $y$ axes. The $X( \pm \pi / 2)$ and $Y( \pm \pi / 2)$ gates require an additional $Z$ rotation of duration $t_{\mathrm{c}}$ that corrects for small parasitic $Z$ evolution during the gate. The $Z$ gates are realized by idling at the avoided crossing for the appropriate fraction of the precession period $1 / t_{\Delta}$. (b) Table of parameters $\varepsilon_{\mathrm{p}}, t_{\mathrm{xy}}, t_{\Delta}$, and $t_{\mathrm{c}}$ for the various $X, Y$, and $Z$ gates, and the calibrated values for CQB-A and CQB-B. (c) Examples of concatenated gates and simulations of the resulting Bloch vector projections on $\left\langle\sigma_{x}\right\rangle,\left\langle\sigma_{y}\right\rangle$, and $\left\langle\sigma_{z}\right\rangle$. (d) Simultaneous randomized benchmarking traces corresponding to Clifford fidelity (errors within the computational subspace) and leakage Clifford fidelity (errors that leave the computational subspace).

in a straightforward and essentially frequency-independent calibration matrix, highlighting a further advantage to eliminating microwave control. The calibration protocol is then to scan the pulse amplitude and frequency to realize high-fidelity single-qubit gates (see Supplemental Material for details of the procedure [31]).

Note that $Z$ gates are realized as idling operations: $Z\left(\phi\left(t_{d}\right)\right)=\exp \left(-i \hat{\sigma}_{z} \phi\left(t_{d}\right) / 2\right)$, where $\phi\left(t_{d}\right)=\Delta t_{d}$ for a gate of duration $t_{d}$, as shown in Fig. 2(a). The gate duration $t_{d}$ determines the type of $Z$ gate along a continua: Increments of quarter periods in the precession period $t_{\Delta} \equiv 2 \pi / \Delta$ at the avoided crossing yield the familiar gates $I, Z( \pm \pi / 2)$, and $Z(\pi)$ [see the table in Fig. 2(b)]. The timing jitter associated with the baseband pulse generator is less than $2 \mathrm{ps}$, compared with the precession period $2 \pi / \Delta \approx 15 \mathrm{~ns}$, corresponding to an error rate of less than $0.02 \%$. This case may be compared with the baseband envelope of a microwave pulse for microwave gates of similar duration.

We use $t_{d}$ as the basic clocking unit for $X( \pm \pi / 2)$ and $Y( \pm \pi / 2)$ gates, compatible with our selected pulse frequency $\omega_{\mathrm{p}} / 2 \pi=125 \mathrm{MHz}$, such that the gates can be completed within the time window and are sufficiently nonadiabatic [see Fig. 2(a)]. The start of the $X( \pm \pi / 2)$ pulse within the window is, in principle, arbitrary, but once chosen, it establishes the $x$ axis for the Bloch sphere. The $y$ axis then corresponds to a $\pi / 2$ phase shift, implemented by advancing the onset of the $Y( \pm \pi / 2)$ gate by an amount $t_{\mathrm{xy}}=t_{\Delta} / 4$, a quarter of the precession period at the avoided crossing. We elect to start the $X( \pm \pi / 2)$ and $Y( \pm \pi / 2)$ pulses symmetrically about the midpoint of the pulse window $t_{d}$, as shown in Fig. 2(a). During the operations, the $X( \pm \pi / 2)$ and $Y( \pm \pi / 2)$ gates may accumulate a small parasitic $Z$ component, which we can correct by padding the gate with corrective $Z$ rotations of duration $t_{\mathrm{c}}$, such that the total duration becomes $t_{d}=t_{\Delta}+t_{\mathrm{c}}$. The calibration parameters for both CQB-A and CQB-B are shown in Fig. 2(b).

We apply these gates to benchmark the coherence properties of the CQB. Within the CQB subspace, the standard coherence metrics are a relaxation time $T_{1}>2 \mathrm{~ms}$, Ramsey time $T_{2 \mathrm{R}} \approx 8 \mu \mathrm{s}$, and Hahn echo time $T_{2 \mathrm{E}} \approx 25 \mu \mathrm{s}$. Monte Carlo simulations of the CQB system are consistent with these times using a noise amplitude of approximately $5 \mu \Phi_{0} / \sqrt{\mathrm{Hz}}$ for each transmon. The long $T_{1}$ time is a general feature of all small-gap qubits [20,40-44], and it can be understood in the context of Fermi's golden rule, where the smaller gap (matrix element that couples the qubit states) translates to a reduced decay rate. In the specific case of excitation or relaxation within the computational subspace of a CQB, a correlated two-photon interaction with the environment is needed, resulting in a relatively low decay rate. Thus, fast, nonadiabatic control is consistent with robust qubit state initialization and operation, despite the presence of a relatively hot environmental bath. Because we can independently read out each individual transmon, we can also extract metrics accounting for leakage to states outside the CQB computational 
subspace (predominantly the ground state $\left|g_{1}, g_{2}\right\rangle$ ). Leakage occurs on a timescale $T_{1, \text { leakage }} \approx 30 \mu \mathrm{s}$ and is comparable with the bare transmon $T_{1}$. While this issue is certainly an area for improvement, error-correction protocols exist to address leakage errors (in any system), and as we describe below, the CQB readout affords an efficient means to detect leakage while protecting the CQB quantum information. The coherence properties of the CQBs and their constituent transmons are tabulated in the Supplemental Material [31].

The gates shown in Fig. 2(a) are concatenated sequentially in a "back-to-back" or "bonded" manner to implement multipulse, nonadiabatic control, realizing encoded operations on the CQB. An example of a sequence of gates is shown in Fig. 2(c), along with numerical simulations of the CQB Bloch vector, to illustrate the operability of this approach. The simulations indicate that high-fidelity universal control is achievable on timescales approaching the inverse coupling strength $1 / \Delta$, which is similar in duration to state-of-the-art single-qubit microwave gates in this test sample and much faster than could be achieved by resonant Rabi driving. The CQB single-qubit gate duration is not limited by the transmon anharmonicity and may therefore be further reduced (baseband pulse generator bandwidth permitting) by increasing $\Delta$. We then obtain the average Clifford fidelity of these nonadiabatic gates using simultaneous randomized benchmarking (RB) on CQB-A and CQB-B, shown in Fig. 2(d). Both Clifford fidelities exceed $99.7 \%$, near state of the art for conventional single-qubit microwave gates $[7,45]$, and they are approximately coherence limited.

\section{B. Noise immunity}

Next, we investigate the CQB susceptibility to various forms of noise via Eq. (1). The CQB is, in principle, linearly sensitive to fluctuations in $\Delta$, but since this frequency is generated predominantly by a lithographically defined, fixed capacitive coupling between transmons, its noise contribution is small. Because of the avoided crossing, the CQB also exhibits the familiar first-order insensitivity to low-frequency magnetic flux noise, which enters via the transverse frequency $\epsilon$. As a result, the CQB flux insensitivity is substantially stronger than that of the individual transmons biased at the corresponding point, $\varphi_{1,2}=\mp \phi *$. The CQB exhibits Hahn echo times exceeding $23 \mu \mathrm{s}$, compared to around $3 \mu \mathrm{s}$ for the individual transmons. Furthermore, the CQB second-order sensitivity to flux noise is inversely proportional to $\Delta$ (the transmontransmon coupling $g$ ) [31]. This result implies that, in addition to enabling faster gates, increasing $\Delta$ will also improve CQB coherence. For the circuit under consideration, the $\Delta / 2 \pi$ that yields an optimal balance between $T_{1}$ and coherence is likely in the low hundreds of $\mathrm{MHz}$.

More substantially, the CQB is first-order insensitive to any such fluctuations in the bare transmon frequencies (i.e.,
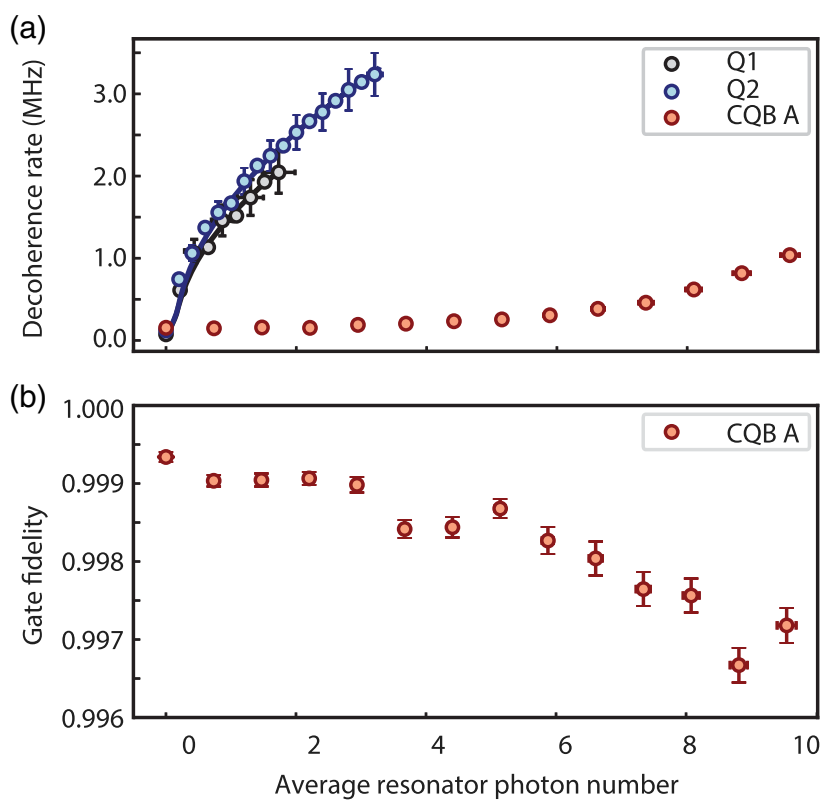

FIG. 3. Photon shot-noise characterization. (a) Ramsey decoherence rate. The CQB is protected by design against photon shot noise, exhibiting a much lower decay rate (see text). In panel (b), we show how this protection extends to operations applied to the encoded qubit, as indicated by the slow falloff of the randomized benchmarking Clifford fidelity with the number of photons in the resonator.

fluctuations in $\epsilon$ ), such as those that arise from photon number fluctuations in the readout resonator. In the dispersive regime, resonator photon fluctuations dephase transmons through an ac Stark shift, which leads to a photon-number-dependent frequency shift $\chi$ of the qubit. The spectrum and amplitude of such photon noise that arises from coherent driving of a resonator is well understood [46]. In Fig. 3(a), the Ramsey decoherence rate as a function of the average number of coherent photons in the resonator of CQB-A is compared with that of its bare transmons. The CQB is substantially less sensitive to these photon number fluctuations compared with the bare transmons, so its coherence is largely preserved.

The CQB insensitivity to photon noise in the resonator implies that the resonator cannot be used for its readout when biased at the avoided level crossing. This case is reminiscent of SQUID-based measurements of persistent current flux qubits biased at degeneracy: Hybridization of the clockwise and counterclockwise circulating currents from strong tunnel coupling prevents a relatively slowreadout SQUID magnetometer from being able to distinguish between the diabatic circulating current states $[34,47]$. Rather, the SQUID is sensitive to the average circulating current in the energy eigenbasis. Similarly, when the tunnel coupling $\Delta$ between the transmons is much stronger than the resonator readout speeds, the resonators are unable to distinguish the diabatic states that hybridize into the CQB subspace. However, importantly, 


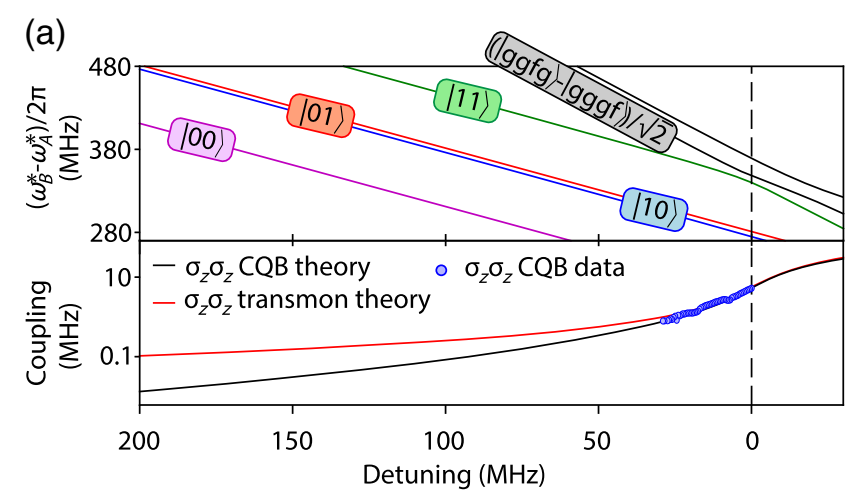

(b)
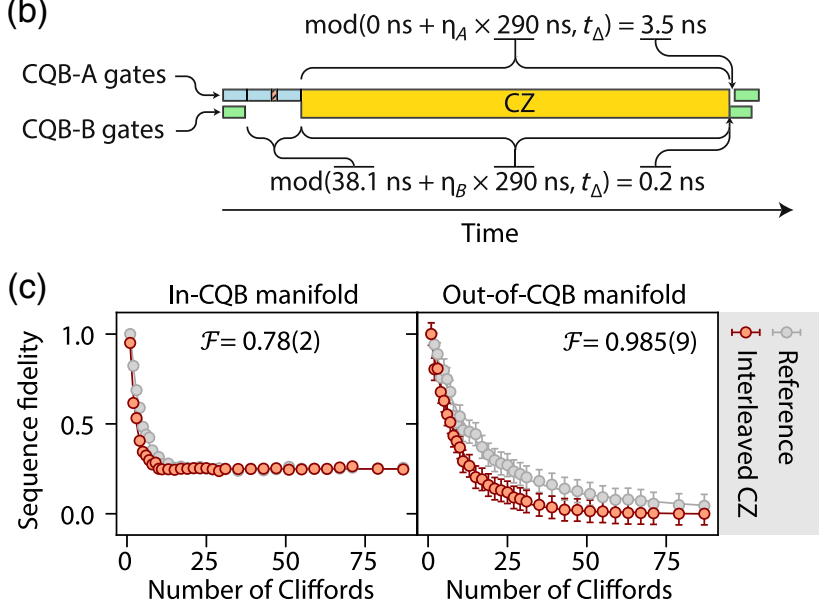

FIG. 4. Two-CQB gates. (a) Controlled-Z (CZ) gate between CQB-A and CQB-B (upper panel), performed by adiabatically ramping the $\mathrm{CQB}$ diabatic frequencies (at the transmon energy degeneracies) $\omega_{\mathrm{A}}^{*}$ and $\omega_{\mathrm{B}}^{*}$ [31], to the avoided level crossing between $|11\rangle$ and the hybridized second excited states $\left(\left|g_{1}, g_{2}, f_{3}, g_{4}\right\rangle-\left|g_{1}, g_{2}, g_{3}, f_{4}\right\rangle\right) / \sqrt{2}$ of CQB-B's bare transmons. The $\mathrm{CQB} \mathrm{CZ}$ avoided level crossing occurs when $\omega_{\mathrm{B}}^{*} \approx \omega_{\mathrm{A}}^{*}+\left(\Delta_{A}+\Delta_{B}\right) / 2+E_{C} / h$. Lower panel: effective $\sigma_{z} \sigma_{z}$ coupling as a function of detuning from the avoided level crossing. (b) Syncing single-CQB gates after a CZ gate, achieved by applying a compensatory $Z$ gate, which takes into account the time of the last single-CQB gate, and phase evolution rate $\eta_{A, B}$ during the CZ. (c) Two-CQB randomized benchmarking within and out of the CQB manifold.

the resonators are capable of discriminating between states that are within and outside the CQB subspace. As a result, the resonators can be continuously monitored to detect leakage without reducing the CQB gate fidelity. We demonstrate this resilience to a continuous readout tone in Fig. 3(b), where the gate fidelities $\mathcal{F}$ remain nearly constant for up to three photons in the resonator. Leakage detection raises the possibility for postselection or error correction on the leakage channel, allowing the $T_{1}$ and $T_{2}$ within the CQB subspace to dictate the operational fidelity (which could, in turn, also be further error corrected in the conventional manner).

\section{Two composite qubit gates}

We complete the universal gate set for quantum computation with a CQB architecture by demonstrating a two$\mathrm{CQB}$ gate. Conventionally, a CZ gate between two transmons is realized by adiabatically tuning one of their frequencies such that its second excited state $\left|g_{1}, f_{2}\right\rangle$ hybridizes with $\left|e_{1}, e_{2}\right\rangle$, inducing a joint $Z Z$ operation [48]. This operation is similarly implemented in our transmon-based CQB architecture by dynamically adjusting $\varphi_{A, B}^{*}$ to hybridize $|11\rangle$ with a noncomputational state, as shown in Fig. 4(a). An important distinction, however, is that the phase between two CQBs can desynchronize from the always-on $Z$ rotations when idling at the avoided crossing. To keep them synchronized, we apply corrective $Z$ operations after each $\mathrm{CZ}$ gate. These corrections are easily computed given the pulse sequence, shown in Fig. 4(b).

During the $\mathrm{CZ}$ gate, the CQBs are kept fully hybridized $\left(\delta f_{\mathrm{A}, \mathrm{B}}=0\right)$ such that they remain insensitive to the frequency fluctuations of their constituent transmons. However, the system becomes sensitive to noise at the relative detuning between the two CQBs during a $\mathrm{CZ}$ gate. Our CZ time was $290 \mathrm{~ns}$ (not including single-qubit gates), corresponding to an optimal interaction time $2 \pi \times 4 / g_{23}=$ 250 ns with additional 20 ns Gaussian ramps to and from the $Z Z$ operation point [Fig. 4(b)]. In conjunction with single-qubit gates, the measured CZ-gate fidelity was $\mathcal{F}=0.77$, obtained by interleaved randomized benchmarking [Fig. 4(c)]. This admittedly moderate fidelity is due in small part to coherence limitations [31], but it is primarily related to an insufficient CZ-gate calibration. In either case, it is not due to a fundamental limitation. The CZ-gate time between two CQBs is increased by a factor of 4 relative to that between two bare transmons with the same coupling strengths. Increasing the coupling between transmons 2 and 3 in Fig. 1(a) will reduce this gate time and thereby improve the error rate [49]. More importantly, we have focused on developing and automating the calibration methods needed to implement high-fidelity $\mathrm{CZ}$ gates with two transmon qubits, having recently achieved two-qubit fidelities of $99.7 \%[7,50]$. With these calibration techniques in place and using optimized device parameters, we expect that twoCQB CZ gates will achieve state-of-the-art fidelities, as its underlying mechanics are nominally identical to a $\mathrm{CZ}$ gate between two bare transmons. In fact, because CQBs are kept at a noise-insensitive point throughout the $\mathrm{CZ}$ operation (unlike $\mathrm{CZ}$ gates with two bare transmons), the CQBs will experience lower dephasing rates during the $\mathrm{CZ}$ gate as compared to performing the same operation in conventional transmon architectures. In conjunction with the higher coherence times within the CQB subspace, this result holds the promise for even higher gate fidelities for CQBs. 


\section{DISCUSSION}

Our results demonstrate that the CQB and other smallgapped qubits can serve as building blocks for quantumcomputing architectures. Using CQBs can reduce sensitivity to many common forms of noise in transmons, including always-on $\sigma_{z} \sigma_{z}$ cross talk with other qubits [see Fig. 4(a), lower panel], allowing for stronger CQBCQB coupling and thereby enabling faster gates in future designs. Furthermore, while transmons are susceptible to two level system appearing near their first-order insensitive point and cannot be detuned without admitting additional flux noise, the transmon frequencies at which the CQB avoided crossing occurs are broadly tunable. While this CQB architecture introduces a source of incoherent leakage out of the computational basis (via relaxation to $\left|g_{1}, g_{2}\right\rangle$ ), we have demonstrated that such leakage can be detected in real time without sacrificing gate fidelity, unlike baretransmon architectures.

More generally, qubits with small gaps-including composite qubits-need not compromise between control speed and protection from decoherence. The nonadiabatic procedures demonstrated here enable the universal control of small-gap systems where conventional Rabi driving is impractical or even infeasible. For example, our demonstration complements a recent parallel work [51] with a small-gap fluxonium qubit [41-43], and it may be useful for other small-gap superconducting qubits such as the metastable flux qubit [40] and the $0-\pi$ qubit [44,52]. Similarly, other systems with small or stable gaps-such as semiconductor-based spin qubits [19,53,54], neutral atomic systems [55], polar molecules [56,57], and laser-dressed NV centers [58] — may also be controllable using these strong driving techniques.

This research was funded in part by the U.S. Army Research Office Grant No. W911NF-18-1-0116 and by the Assistant Secretary of Defense for Research \& Engineering via MIT Lincoln Laboratory under Air Force Contract No. FA8721-05-C-0002. D. L. C. gratefully acknowledges support by an appointment to the Intelligence Community Postdoctoral Research Fellowship Program at the Massachusetts Institute of Technology, administered by Oak Ridge Institute for Science and Education through an interagency agreement between the U.S. Department of Energy and the Office of the Director of National Intelligence. B. K. gratefully acknowledges support from the National Defense Science and Engineering Graduate Fellowship program.

[1] J. Koch, T. M. Yu, J. Gambetta, A. A. Houck, D. I. Schuster, J. Majer, A. Blais, M. H. Devoret, S. M. Girvin, and R. J. Schoelkopf, Charge-Insensitive Qubit Design Derived from the Cooper Pair Box, Phys. Rev. A 76, 042319 (2007).
[2] F. Yan, S. Gustavsson, A. Kamal, J. Birenbaum, A. P. Sears, D. Hover, T. J. Gudmundsen, D. Rosenberg, G. Samach, S. Weber, J. L. Yoder, T. P. Orlando, J. Clarke, A. J. Kerman, and W. D. Oliver, The Flux Qubit Revisited to Enhance Coherence and Reproducibility, Nat. Commun. 7, 12964 (2016).

[3] M. H. Devoret and R. J. Schoelkopf, Superconducting Circuits for Quantum Information: An Outlook, Science 339, 1169 (2013).

[4] W. D. Oliver and P. B. Welander, Materials in Superconducting Quantum Bits, MRS Bull. 38, 816 (2013).

[5] J. M. Gambetta, J. M. Chow, and M. Steffen, Building Logical Qubits in a Superconducting Quantum Computing System, npj Quantum Inf. 3, 2 (2017).

[6] G. Wendin, Quantum Information Processing with Superconducting Circuits: A Review, Rep. Prog. Phys. 80, 106001 (2017).

[7] M. Kjaergaard, M. E. Schwartz, J. Braumller, P. Krantz, J. I.-J. Wang, S. Gustavsson, and W. D. Oliver, Superconducting Qubits: Current State of Play, Annu. Rev. Condens. Matter Phys. 11, 369 (2020).

[8] P. Krantz, M. Kjaergaard, F. Yan, T. P. Orlando, S. Gustavsson, and W.D. Oliver, A Quantum Engineer's Guide to Superconducting Qubits, Appl. Phys. Rev. 6, 021318 (2019).

[9] F. Arute et al., Quantum Supremacy Using a Programmable Superconducting Processor, Nature (London) 574, 505 (2019).

[10] M. Kjaergaard et al., A Quantum Instruction Set Implemented on a Superconducting Quantum Processor, arXiv:2001.08838.

[11] F. H. L. Koppens, C. Buizert, K. J. Tielrooij, I. T. Vink, K. C. Nowack, T. Meunier, L. P. Kouwenhoven, and L. M. K. Vandersypen, Driven Coherent Oscillations of a Single Electron Spin in a Quantum Dot, Nature (London) 442, 766 (2006).

[12] M. Veldhorst, J. C. C. Hwang, C. H. Yang, A. W. Leenstra, B. de Ronde, J. P. Dehollain, J. T. Muhonen, F. E. Hudson, K. M. Itoh, A. Morello, and A. S. Dzurak, An Addressable Quantum Dot Qubit with Fault-Tolerant Control-Fidelity, Nat. Nanotechnol. 9, 981 (2014).

[13] D. P. DiVincenzo, D. Bacon, J. Kempe, G. Burkard, and K. B. Whaley, Universal Quantum Computation with the Exchange Interaction, Nature (London) 408, 339 (2000).

[14] D. Bacon, J. Kempe, D. A. Lidar, and K. B. Whaley, Universal Fault-Tolerant Quantum Computation on Decoherence-Free Subspaces, Phys. Rev. Lett. 85, 1758 (2000).

[15] J. Kempe, D. Bacon, D. A. Lidar, and K. B. Whaley, Theory of Decoherence-Free Fault-Tolerant Universal Quantum Computation, Phys. Rev. A 63, 042307 (2001).

[16] Y. Nakamura, Y. A. Pashkin, and J.S. Tsai, Coherent Control of Macroscopic Quantum States in a SingleCooper-Pair Box, Nature (London) 398, 786 (1999).

[17] Y. A. Pashkin, T. Yamamoto, O. Astafiev, Y. Nakamura, D. V. Averin, and J. S. Tsai, Quantum Oscillations in Two Coupled Charge Qubits, Nature (London) 421, 823 (2003).

[18] T. Yamamoto, Y. A. Pashkin, O. Astafiev, Y. Nakamura, and J.S. Tsai, Demonstration of Conditional Gate Operation 
Using Superconducting Charge Qubits, Nature (London) 425, 941 (2003).

[19] Y.-P. Shim and C. Tahan, Semiconductor-Inspired Design Principles for Superconducting Quantum Computing, Nat. Commun. 7, 11059 (2016).

[20] W. D. Oliver, Y. Yu, J. C. Lee, K. K. Berggren, L. S. Levitov, and T.P. Orlando, Mach-Zehnder Interferometry in a Strongly Driven Superconducting Qubit, Science 310, 1653 (2005).

[21] M. Sillanpää, T. Lehtinen, A. Paila, Y. Makhlin, and P. Hakonen, Continuous-Time Monitoring of Landau-Zener Interference in a Cooper-Pair Box, Phys. Rev. Lett. 96, 187002 (2006).

[22] S. Shevchenko, S. Ashhab, and F. Nori, Landauzenerstückelberg Interferometry, Phys. Rep. 492, 1 (2010).

[23] D. Vion, A. Aassime, A. Cottet, P. Joyez, H. Pothier, C. Urbina, D. Esteve, and M. Devoret, Manipulating the Quantum State of an Electrical Circuit, Science 296, 886 (2002).

[24] Y. Yoshihara, K. Harrabi, A. Niskanen, Y. Nakamura, and J. Tsai, Manipulating the Quantum State of an Electrical Circuit, Phys. Rev. Lett. 97, 167001 (2006).

[25] J. Bylander, S. Gustavsson, F. Yan, F. Yoshihara, K. Harrabi, G. Fitch, D. G. Cory, Y. Nakamura, J.-S. Tsai, and W. D. Oliver, Noise Spectroscopy through Dynamical Decoupling with a Superconducting Flux Qubit, Nat. Phys. 7, 565 (2011).

[26] D. I. Schuster, A. Wallraff, A. Blais, L. Frunzio, R.-S. Huang, J. Majer, S. M. Girvin, and R. J. Schoelkopf, ac Stark Shift and Dephasing of a Superconducting Qubit Strongly Coupled to a Cavity Field, Phys. Rev. Lett. 94, 123602 (2005).

[27] A. P. Sears, A. Petrenko, G. Catelani, L. Sun, H. Paik, G. Kirchmair, L. Frunzio, L. I. Glazman, S. M. Girvin, and R. J. Schoelkopf, Photon Shot Noise Dephasing in the Strong-Dispersive Limit of Circuit QED, Phys. Rev. B 86, 180504 (2012).

[28] F. Yan, D. Campbell, P. Krantz, M. Kjaergaard, D. Kim, J. L. Yoder, D. Hover, A. Sears, A. J. Kerman, T. P. Orlando, S. Gustavsson, and W. D. Oliver, Distinguishing Coherent and Thermal Photon Noise in a Circuit Quantum Electrodynamical System, Phys. Rev. Lett. 120, 260504 (2018).

[29] M. D. Hutchings, J. B. Hertzberg, Y. Liu, N. T. Bronn, G. A. Keefe, M. Brink, J. M. Chow, and B. L. T. Plourde, Tunable Superconducting Qubits with Flux-Independent Coherence, Phys. Rev. Applied 8, 044003 (2017).

[30] R. Barends, J. Kelly, A. Megrant, D. Sank, E. Jeffrey, Y. Chen, Y. Yin, B. Chiaro, J. Mutus, C. Neill, P. OMalley, P. Roushan, J. Wenner, T. C. White, A. N. Cleland, and J. M. Martinis, Coherent Josephson Qubit Suitable for Scalable Quantum Integrated Circuits, Phys. Rev. Lett. 111, 080502 (2013).

[31] See Supplemental Material at http://link.aps.org/ supplemental/10.1103/PhysRevX.10.041051 for supporting text and figures related to the composite qubit state preparation, characterization, measurement, control, and noise immunity.

[32] A. L. Gramajo, D. Campbell, B. Kannan, D. K. Kim, A. Melville, B. M. Niedzielski, J. L. Yoder, M. J. Sánchez, D. Domínguez, S. Gustavsson, and W. D. Oliver, Quantum
Simulation of Coherent Backscattering in a System of Superconducting Qubits, arXiv:1912.12488.

[33] J. E. Mooij, T. P. Orlando, L. Levitov, L. Tian, C. H. van der Wal, and S. Lloyd, Josephson Persistent-Current Qubit, Science 285, 1036 (1999).

[34] T. P. Orlando, J. E. Mooij, L. Tian, C. H. van der Wal, L. S. Levitov, S. Lloyd, and J. J. Mazo, Superconducting Persistent-Current Qubit, Phys. Rev. B 60, 15398 (1999).

[35] D. M. Berns, W. D. Oliver, S. O. Valenzuela, A. V. Shytov, K. K. Berggren, L. S. Levitov, and T. P. Orlando, Coherent Quasiclassical Dynamics of a Persistent Current Qubit, Phys. Rev. Lett. 97, 150502 (2006).

[36] S. O. Valenzuela, W. D. Oliver, D. M. Berns, K. K. Berggren, L. S. Levitov, and T. P. Orlando, MicrowaveInduced Cooling of a Superconducting Qubit, Science 314, 1589 (2006).

[37] D. M. Berns, M. S. Rudner, S. O. Valenzuela, K. K. Berggren, W. D. Oliver, L. S. Levitov, and T. P. Orlando, Amplitude Spectroscopy of a Solid-State Artificial Atom, Nature (London) 455, 51 (2008).

[38] J. Bylander, M. S. Rudner, A. V. Shytov, S. O. Valenzuela, D. M. Berns, K. K. Berggren, L. S. Levitov, and W. D. Oliver, Pulse Imaging and Nonadiabatic Control of Solid-State Artificial Atoms, Phys. Rev. B 80, 220506(R) (2009).

[39] S. Gustavsson, J. Bylander, and W. D. Oliver, Time-Reversal Symmetry and Universal Conductance Fluctuations in a Driven Two-Level System, Phys. Rev. Lett. 110, 1 (2013).

[40] A. J. Kerman, Metastable Superconducting Qubit, Phys. Rev. Lett. 104, 027002 (2010).

[41] V. E. Manucharyan, J. Koch, L. I. Glazman, and M. H. Devoret, Fluxonium: Single Cooper-Pair Circuit Free of Charge Offsets, Science 326, 113 (2009).

[42] I. M. Pop, K. Geerlings, G. Catelani, R. J. Schoelkopf, L. I. Glazman, and M. H. Devoret, Coherent Suppression of Electromagnetic Dissipation Due to Superconducting Quasiparticles, Nature (London) 508, 369 (2014).

[43] L. B. Nguyen, Y.-H. Lin, A. Somoroff, R. Mencia, N. Grabon, and V.E. Manucharyan, High-Coherence Fluxonium Qubit, Phys. Rev. X 9, 041041 (2019).

[44] A. Gyenis, P. S. Mundada, A. D. Paolo, T. M. Hazard, X. You, D. I. Schuster, J. Koch, A. Blais, and A. A. Houck, Experimental Realization of an Intrinsically ErrorProtected Superconducting Qubit, arXiv:1910.07542.

[45] R. Barends, J. Kelly, A. Megrant, A. Veitia, D. Sank, E. Jeffrey, T. C. White, J. Mutus, A. G. Fowler, B. Campbell, Y. Chen, Z. Chen, B. Chiaro, A. Dunsworth, C. Neill, P. O'Malley, P. Roushan, A. Vainsencher, J. Wenner, A. N. Korotkov, A. N. Cleland, and J. M. Martinis, Superconducting Quantum Circuits at the Surface Code Threshold for Fault Tolerance, Nature (London) 508, 500 (2014).

[46] J. Gambetta, A. Blais, D. I. Schuster, A. Wallraff, L. Frunzio, J. Majer, M. H. Devoret, S. M. Girvin, and R. J. Schoelkopf, Qubit-Photon Interactions in a Cavity: Measurement-Induced Dephasing and Number Splitting, Phys. Rev. A 74, 042318 (2006).

[47] I. Chiorescu, Y. Nakamura, C. J. P. M. Harmans, and J. E. Mooij, Coherent Quantum Dynamics of a Superconducting Flux Qubit, Science 299, 1869 (2003). 
[48] L. DiCarlo, J. M. Chow, J. M. Gambetta, L. S. Bishop, B. R. Johnson, D. I. Schuster, J. Majer, A. Blais, L. Frunzio, S. M. Girvin, and R. J. Schoelkopf, Demonstration of Two-Qubit Algorithms with a Superconducting Quantum Processor, Nature (London) 460, 240 (2009).

[49] J. M. Martinis and M. R. Geller, Fast Adiabatic Qubit Gates Using Only $\sigma_{z}$ Control, Phys. Rev. A 90, 022307 (2014).

[50] M. Kjaergaard et al., A Quantum Instruction Set Implemented on a Superconducting Quantum Computer, arXiv: 2001.08838.

[51] H. Chang, S. Chakram, T. Roy, N. Earnest, Y. Lu, Z. Huang, D. Weiss, J. Koch, and D. I. Schuster, Universal Fast Flux Control of a Coherent, Low-Frequency Qubit, arXiv: 2002.10653.

[52] P. Brooks, A. Kitaev, and J. Preskill, Protected Gates for Superconducting Qubits, Phys. Rev. A 87, 052306 (2013).

[53] J. R. Petta, H. Lu, and A. C. Gossard, A Coherent Beam Splitter for Electronic Spin States, Science 327, 669 (2010).

[54] R. W. Andrews, C. Jones, M. D. Reed, A. M. Jones, S. D. Ha, M. P. Jura, J. Kerckhoff, M. Levendorf, S. Meenehan,
S. T. Merkel, A. Smith, B. Sun, A. J. Weinstein, M. T. Rakher, T. D. Ladd, and M. G. Borselli, Quantifying Error and Leakage in an Encoded $\mathrm{Si} / \mathrm{SiGe}$ Triple-Dot Qubit, Nat. Nanotechnol. 14, 747 (2019).

[55] H. Bernien, S. Schwartz, A. Keesling, H. Levine, A. Omran, H. Pichler, S. Choi, A. S. Zibrov, M. Endres, M. Greiner et al., Probing Many-Body Dynamics on a 51-Atom Quantum Simulator, Nature (London) 551, 579 (2017).

[56] R. Côté, S. Ylein, and D. DeMille, Quantum Information Processing with Ultracold Polar Molecules, in Cold Molecules: Theory, Experiment, Applications, edited by R. V. Krems, W. D. Stwalley, and B. Friedrich (CRC Press, Boca Raton, FL, 2009).

[57] P. Yu, L. W. Cheuk, I. Kozyryev, and J. M. Doyle, A Scalable Quantum Computing Platform Using SymmetricTop Molecules, New J. Phys. 21, 093049 (2019).

[58] C. Avinadav, R. Fischer, P. London, and D. Gershoni, TimeOptimal Universal Control of Two-Level Systems under Strong Driving, Phys. Rev. B 89, 245311 (2014). 